\title{
A novel host-vector system for Butyrivibrio fibrisolvens and its possible use for developing more fibrolytic rumen bacteria
}

\author{
Y Kobayashi, N Okuda, M Wakita, S Hoshino \\ Facuity of Bioresources, Mie University, Tsu, Mie 514, Japan
}

It has been considered as a main target in gene modification of rumen bacteria to enhance their cellulolytic and hemicellulolytic activities. However, no significant progress has been made be cause of the lack of effective gene transfer systems for rumen bacteria. We have embarked on a project to develop a hostvector system for Butyrivibrio fibrisolvens, a predominant xylanolytic bacterium in the rumen, and to modify the species into a more fibrolytic bacterium by heterologous gene expression.

A cryptic plasmid pRJF2 was isolated from a deer rumen bacterium $B$. fibrisolvens $\mathrm{OB} 157$ and sequenced. Its size was 2971 bp with a region appearing to control its replication (1.8 $\mathrm{kb})$. Shuttle vectors, pYK2 (4.4 kb) and pYK3 $(6.1 \mathrm{~kb})$, for transfering DNA between $E$. coli and $B$. fibrisolvens were constructed by ligating the replication region of $\mathrm{pRJF} 2$ into $\mathrm{pUC} 18$ and pBR322, respectively. A fragment containing erm from $\mathrm{pAM} \beta 1$ was inserted into $\mathrm{pYK} 2$ as a selectable marker to construct another shuttle vector pYK4 $(7.9 \mathrm{~kb})$. These vectors harvested from $E$. coli were used to transform $B$. fibrisolvens OB 157 and another strain OB156 by electroporation. erm was expressed well in B. fibrisolvens OB156 but not in OB157, while amp and tet from pUC18 and/or pBR322 were not expressed in both the hosts. This shows that pYK4 and $B$. fibrisolvens OB156 are available for transforming $B$. fibrisolvens.

A Eubacterium ruminantium xylanase gene previously cloned and sequenced was used as target gene for amplification and expression in $B$. fibrisolvens. The gene was truncated in its $\mathrm{C}$-terminus, fused with a stop codon linker and ligated into $P Y K 4$. The resultant chimeric plasmid $\mathrm{pYK} 4 \mathrm{X}$ was electroporated into $B$. fibrisolvens OB156. Transformants were screened for erm expression, elevated xylanolysis, zymogram and southern analysis. No significant change in growth rate on xylan was observed after the recombination. However, an increased accumulation of reducing sugars was noted in the medium of the recombinant $B$. fibrisolvens. Agarose gel electrophoresis showed the recombinant to have a high copy number of the target gene, while the xylanolytic activity was elevated 2-3 times. Xylanolysis by both the recombinant and parent strains was repressed by glucose, cellobiose or xylose, though xylanolysis elevation (2-3 fold) did not change. An alternative promotor for expression of this foreign xylanase gene, proteolysis of the translated product and for regulating xylanase production in originally xylanolytic $B$. fibrisolvens should be explored to promote higher and more stable gene expression. 